\title{
Entretien
}

\section{Robert Barbault ou de l'écologie à la gestion de la biodiversité}

\section{Propos recueillis par Pierre Migot et Marie Roué ${ }^{1}$}

\author{
Robert Barbault ${ }^{a}$ Pierre Migot ${ }^{b}$, Marie Rouéc \\ a Écologue, Muséum national d'histoire naturelle, Département Écologie et gestion de la biodiversité, \\ 36 rue Geoffroy Saint-Hilaire, 75231 Paris Cedex 05 \\ b Biologiste, Office national de la chasse et de la faune sauvage, Direction des études et de la recherche, BP 20, \\ 78612 Le Perray en Yvelines Cedex \\ c Anthropologue, Muséum national d'histoire naturelle Département Hommes, natures, sociétés, BP 26, 57 rue Cuvier, \\ 75231 Paris Cedex 05
}

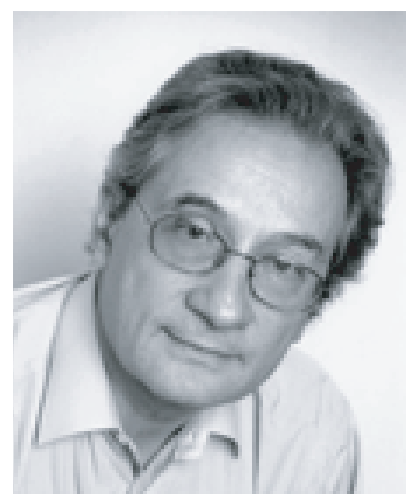

Pierre Migot : La gestion des ressources naturelles s'appuie sur l'écologie de la conservation, expression que vous utilisez dans votre ouvrage à l'intention des étudiants $^{2}$ qui, dites-vous, est une synthèse de nombreuses sciences de base tant dans le domaine des sciences biologiques, que dans le domaine des sciences humaines. Quelles sont les idées nouvelles et les approches apportées par cette démarche de synthèse? Quels sont selon vous les axes de recherche à développer?

Robert Barbault : Sur l'écologie de la conservation comme science de synthèse, je ne suis pas convaincu qu'il

\footnotetext{
Auteur correspondant : R. Barbault, barbault@mnhn.fr

${ }^{1}$ Remerciement à Brigitte Iwach (ONCFS) pour la transcription de l'enregistrement de l'entretien.

2 Barbault, R., 1997, Biodiversité, Paris, Hachette, la bibliothèque de base de l'étudiant en sciences.
}

faille voir les choses exactement comme cela. C'est peutêtre la prétention, ou l'objectif, ce à quoi aspire cette nouvelle configuration d'une partie de l'écologie regroupée avec la génétique des populations, mais elle est loin de correspondre à une synthèse des sciences en question. Les initiateurs de cette dynamique, qui étaient à l'origine plus des écologues que des généticiens, avaient compris que pour atteindre leurs objectifs, pour mettre en œuvre la conservation de la nature, pour en faire une science d'action qui ne se contente pas de «qu'est-ce qu'il faudrait faire?», mais qui s'implique, cette discipline avait besoin d'autres compétences. Mais ces disciplines, ou leurs acteurs, n'ont pas été invités au débat, n'ont pas participé à une élaboration conceptuelle commune.

Dans l'action, ces biologistes collaborent avec des collègues $\mathrm{d}$ 'autres origines, mais je crois que cette science, en tant que champ interdisciplinaire, reste à construire, à mûrir, à travers précisément les défis posés par l'action. Ce que j'ai vu de nouveau dans le phénomène, c'est l'exigence d'une construction théorique. Mais en ce qui concerne la mobilisation des autres compétences, elles l'étaient déjà avant, et là, je ne suis pas sûr que cette façon de positionner les choses ait fait sensiblement avancer l'interdisciplinarité. Quand je lis ces auteurs, Michaël Soulé ou d'autres, je trouve que c'est une pensée d'écologues engagés qui mobilise d'autres personnes, sans que cela soit vraiment totalement partagé.

Marie Roué : Les bons sentiments sur le bon usage de la nature sont communs, mais il est plus difficile de 


\begin{abstract}
Biographie
Robert Barbault, docteur ès sciences, est professeur à l'université Paris-VI, directeur de l'Institut fédératif de recherche «Écologie : biodiversité, évolution, environnement » (IFR 101), et directeur du département Écologie et gestion de la biodiversité du Muséum national d'histoire naturelle.

Il est président du Comité français du programme l'Homme et la biosphère de l’Unesco, et membre du Conseil scientifique du patrimoine naturel et de la biodiversité placé auprès du ministre chargé de l’Environnement. Il préside le Conseil scientifique du Cemagref.

Ses recherches portent sur la biologie des populations et l'écologie, et notamment la dynamique des populations de vertébrés, l'organisation des peuplements, la biodiversité et la biologie de la conservation. Il est l'auteur de nombreuses publications dans des revues scientifiques à audience internationale. Il a participé à la rédaction de chapitres de près d'une vingtaine d'ouvrages d'audience internationale. Il a publié plusieurs livres au cours des dernières années : Des bactéries et des hommes, Ed. Odile Jacob, 1994 ; La révolution technologique en écologie (sous la direction de J.-M. Legay et R. Barbault), Ed. Masson, 1995 ; Écologie générale - structure et fonctionnement de la biosphère, Ed. Dunod, 2003 ; Biodiversité et changements globaux (sous la direction de R. Barbault et B. Chevassus-au-Louis), adpf. ministère des Affaires étrangères 2004; Un éléphant dans un jeu de quilles - l'Homme dans la biodiversité, Ed. du Seuil, 2006.

Il a reçu le Prix Pouchard de l'Académie des Sciences (1983), est chevalier de l'ordre national du Mérite et de l'ordre du Mérite Agricole et membre de l'Academia Europaea.
\end{abstract}

transformer radicalement les visions du monde des uns et des autres. Le fait que la vision du monde des sciences de la société diverge de celle des sciences de la nature, sans parler des différences de vue entre différentes disciplines à l'intérieur des sciences de la nature, pose problème.

Robert Barbault : Oui, construire une interdisciplinarité opérationnelle par rapport à ces objectifs-là, ça ne se décrète pas. Mais, quand on regarde ce domaine de mobilisation des gens autour de la nature ou de la conservation de la nature, ça a quand même beaucoup progressé, pas seulement parce qu'il y a eu une science qui s'est baptisée "Conservation Biology». C'est un mouvement d'ensemble. Ainsi, quand on lit certains documents patronnés par l'UICN (Union mondiale pour la nature), ou par le WWF (World Wildlife Fund) ou d'autres, on voit que les grandes ONG internationales, avaient déjà fait des percées importantes, qui étaient à la limite peut être plus avancées, plus équilibrées que ce qu'on trouvait dans les manuels de biologie de la conservation des années 1980-1990.

D'ailleurs, si j'utilise de préférence l'expression « écologie de la conservation », c'est parce que l'expression «biologie de la conservation » me met mal à l'aise. Quand on enseigne, on est bien obligé de dire que ce n'est pas de la «biologie de la conservation », mais que c'est plutôt une composante des sciences de la conservation, qui s'enracine dans la biologie et l'écologie, et qui réalise que les problèmes ne sont pas strictement biologiques, ou pas nécessairement biologiques dans certains cas. Baptiser ça «biologie de la conservation » cela met tout par terre et nuit à la communication. En tout cas au niveau français, ça ne favorise pas précisément les échanges interdisciplinaires que l'on souhaiterait avoir, parce que ça fait quand même monopole, impérialisme. Que cela ait été voulu ou non, les effets sont garantis!

P.M. : En fait, d'après ce que vous dites ou d'après ce que vous avez écrit, c'est l'ensemble des sciences qui contribuent à la conservation. Quelles seraient les démarches de recherche les plus pertinentes pour contribuer à la conservation de la biodiversité? J'ai retenu deux approches : d'abord la recherche-observation, avec la description des phénomènes et leur suivi spatiotemporel, qui permet à l'ensemble des acteurs de prendre acte de la situation biologique et ensuite la dimension « recherche-action », avec l'utilisation des disciplines spécialisées, dans le cadre des relations entre chercheurs et gestionnaires, qui permet de trouver une solution et le cas échéant de valider la pertinence des propositions de gestion.

Robert Barbault : Je suis tout à fait d'accord avec ça, et à la limite on peut s'interroger sur le fait : «Est-ce que cette biologie de la conservation est vraiment une science? », «Est-ce qu'on a besoin d'une science qui se baptise comme ça? » Il y a des objectifs de conservation, de gestion de la nature, d'aménagement du territoire par rapport à des préoccupations de conservation, mais est ce que cela a un sens de dire qu'il y a " une science » au singulier de la chose? On peut dire que c'est une question de vocabulaire. Si on dit «sciences de la conservation » au pluriel, comme on en a pris l'habitude maintenant, notamment lors de colloques, c'est en réponse à ce malaise. Si on met «écologie de la conservation » ça peut corriger le tir un petit peu, à condition que tout le monde soit d'accord sur le fait que c'est une écologie qui a évolué, qui revient à ses racines et qui est très interdisciplinaire, ce que j'appelle une vision écologique du monde dans laquelle on inclura les sociétés humaines, mais cela n'est pas forcément partagé non plus. Avec en plus l'ambiguïté entre écologie militante et écologie scientifique, ça ne résout pas vraiment le problème. En disant «sciences de la conservation ", ce n'est certes pas fascinant, mais c'est quand même la réalité : c'est l'ensemble des sciences, des techniques, des compétences, des pratiques, des usages qui sont mobilisés. 
P.M. : Dans un texte récent ${ }^{3}$ vous soulignez le saut majeur franchi avec la publication de la Stratégie mondiale de la conservation publiée en 1992 sous l'égide du World Ressources Institute, de la World Conservation Union et de 1'UNEP (United nations Environment Programme) qui précise qu'il faut protéger le fonctionnement et la diversité des systèmes naturels, non seulement pour leur valeur intrinsèque, mais aussi pour le développement durable auquel ont droit les populations humaines. Comme Catherine et Raphaël Larrère ${ }^{4}$ le disent également, si la conservation de la biodiversité ne se réduit plus à la protection des espèces sauvages dans des réserves naturelles, il ne s'agit donc plus de geler une nature sauvage, mais de préserver la capacité évolutive des processus écologiques, d'assurer une gestion complexe d'espaces diversifiés.

Pour l'écologue que vous êtes, qu'est-ce que cette nouvelle donne change à vos objectifs et à votre pratique scientifique?

Robert Barbault : Pour moi, le changement a été progressif. J'ai réalisé que les questions de conservation de la nature se plaçaient dans le cadre que nous venons d'évoquer, et que cela avait des conséquences importantes sur l'ensemble des représentations qu'on en avait, des priorités qu'il fallait mettre en œuvre. Et donc cela a abouti à un glissement progressif vers des questions touchant à la conservation de la nature. Mais, plus que cela, je me suis intéressé surtout aux relations entre la science et la société, et cela progressivement. Quand j'ai commencé à me pencher sur les "réserves de biosphère », alors que 1'Unesco m'envoyait en mission au Mexique, je n'étais qu'un écologue de base qui s'intéressait à la dynamique des populations et aux mécanismes de régulation des populations animales. Je découvrais alors le programme MAB (Man and Biosphere) de l'Unesco. Je n'en avais pas une forte opinion, mais c'était encore le début et je n'en mesurais pas toute la portée. Ce que je voyais, c'était l'opportunité de travailler, de faire des expertises, au Mexique après l'avoir fait en Afrique. Ayant de plus des affinités avec la langue espagnole, je ne pouvais rêver mieux. Puis, j'ai découvert petit à petit, au fil des missions, ce qu'était cette réserve de biosphère dans le désert du Chihuahua au Nord du Mexique. J'ai constaté que cet immense espace mis en réserve était en grande partie la propriété d'une seule famille. Certes, il s'agit de zones désertiques avec des pâturages naturels et un élevage extensif, mais c'est là où j'ai commencé à $\mathrm{m}^{\prime}$ interroger sur les motivations des gens. Pourquoi un grand propriétaire souhaite bénéficier du label « Réserve

\footnotetext{
${ }^{3}$ Génot, J.-C., Barbault, R., 2004. Quelle politique de conservation? in Barbault, R. et Chevassus-au-Louis, B. (Eds.), Biodiversité et changements globaux, Paris, ADPF, ministère des Affaires étrangères, p. 162-191

${ }^{4}$ Larrère, C., Larrère, R., 1997. Du bon usage de la nature - Pour une philosophie de l'environnement, Paris, Aubier.
}

de biosphère » et accepte d'entrer dans ce processus ? J'ai compris assez rapidement, à travers des discussions avec d'autres personnes de la région sur l'évolution de la politique agricole au Mexique, les problèmes de gestion de la terre, de réforme agraire. Manifestement, il y avait un risque, certes faible, que l'on aboutisse à des décisions du genre : il faut répartir l'exploitation de ces vastes espaces entre les différents utilisateurs (les vaqueros employés et vivant sur ces terres), avec de gros risques de surpâturage, car ce qui est possible sur une vaste superficie ne l'est pas sur des petites propriétés. Ainsi, le propriétaire en question a vu son intérêt. C'était pour lui une façon de sauvegarder ses propres conditions de vie, tout en s'impliquant dans le processus de conservation de la biodiversité, en acceptant que les chercheurs viennent, que des zones soient en protection intégrale et qu'en même temps, on se préoccupe de développement durable. Tout cela n'a pas eu pour conséquence de me faire évoluer sur un plan scientifique proprement dit, mais à m'intéresser de moins en moins exclusivement à la recherche proprement dite, et davantage aux conditions de la recherche, à la gestion de la recherche.

Cela s'est fait par une suite de hasards, lorsque l'Unesco m'a envoyé au Mexique, comme quand on m'a demandé d'accepter la responsabilité de chargé de mission au CNRS, et donc de m'impliquer dans la politique scientifique. Je n'y avais pas réfléchi avant, je ne m’étais jamais dit: "Moi, la politique scientifique, ça m'intéresse», de même que je ne m'étais jamais dit : "Je veux diriger un laboratoire. » On m'aurait posé la question, j'aurais dit que je n'étais pas fait pour ça, que je n'avais pas la personnalité qui convient. Puis de fait, j'ai fait toutes ces choses-là, car précisément derrière il y avait une certaine «écologie humaine »; je me suis aperçu que ça concernait les activités des gens, collègues, étudiants, et c'est à travers la dimension humaine de ces choses que je me suis intéressé à ce type d'activités.

Pour le programme MAB, ça a été la même chose. Réintroduire les sociétés humaines par rapport à des préoccupations d'écologie était important pour moi. Pour dire la vérité d'ailleurs, j'en avais pris conscience déjà quand j'étais en Côte-d'Ivoire, quand je faisais des recherches sur la dynamique des populations de lézards. Je travaillais avec des Baoulés, et ils essayaient de comprendre pourquoi on faisait ça. Je dois dire que je souffrais un peu lorsque je devais leur expliquer ce que l'on faisait, quel en était le sens. J'avais du mal à montrer en quoi le fait de s'intéresser aux mécanismes de régulation des populations de lézards des savanes de Côte-d'Ivoire, pouvait avoir une importance pour les populations humaines qui y vivent. J'avais donc un problème par rapport à mon activité scientifique, j'avais conscience de son faible intérêt par rapport à de grandes questions de société. Je pouvais, certes tenir un discours expliquant les liens entre régulation des populations animales et équilibre de 
la nature, etc. Mais je pensais qu'il eut été plus intéressant de le faire sur des rivières, des lacs, des espaces où l'homme a des intérêts plus forts.

L'évolution vers les concepts de biodiversité et de développement durable, s'est inscrite dans cette perspective. Au début, quand Fransesco Di Castri m'a parlé de biodiversité, je n'ai pas rigolé, mais j'ai pensé «la diversité du vivant, il n'y a rien de nouveau sur la planète ». Mais il savait susciter l'enthousiasme, et de même qu'il m'avait entraîné dans les réserves de biosphère au Mexique, je l'ai suivi sur ce nouveau terrain. Cela a commencé à Rio avec des débats sur la biodiversité. Ce que j'ai découvert - et ce qui m’a intéressé, c'était les questionnements sur la diversité du vivant; les liens entre diversité du vivant et l'homme : cela ouvrait complètement le champ à des approches tout à fait différentes, nouvelles.

M.R. : Vous êtes président du comité français MAB de l'Unesco et donc à ce titre, vous vous êtes particulièrement intéressé aux réserves de la biosphère, comme vous venez déjà de le souligner. Mais les enjeux actuels, ne sont-ils pas de passer de la protection d'espaces particuliers, parfois mis sous cloche, à une gestion intégrée de la biodiversité sur l'ensemble de la planète dans une perspective de développement durable? Dans cette perspective, comment voyez-vous l'évolution du concept « réserves de biosphère » ? Faut-il donner plus de place aux sciences humaines dans une vision où développement durable et conservation vont de pair? Et si oui, comment l'envisagez-vous?

Robert Barbault : Le concept de réserve de biosphère et la mise en œuvre des réserves de biosphère étaient déjà une anticipation des préoccupations actuelles sur le développement durable. Je pense que les premiers sites qui ont été délimités, ne l'ont pas été pour les mettre sous cloche. C'est clair, énoncé dès l'origine. Les initiateurs étaient généralement des écologues, mais qui avaient fait le constat que pour aller plus loin dans la compréhension de la dynamique du vivant, dans la compréhension des écosystèmes, il fallait impliquer les sociétés humaines, il fallait se préoccuper de leur développement. Et donc, l'Unesco a eu l'idée de lancer ce programme MAB, «l'Homme et la biosphère », et de l'incarner dans des espaces, et justement dans des espaces habités. Les propositions concrètes de réserves de biosphère devaient émaner des populations concernées, c'est-à-dire, qu'en aucun cas cela ne pouvait être une décision, ni de l'Unesco à Paris, ni du gouvernement du pays concerné. Le gouvernement devait transmettre à l’Unesco la proposition, l'Unesco respectant les prérogatives de chaque État, et c'était un projet qui devait être préparé par l'ensemble des acteurs du territoire. Ainsi, c'est ce que j'ai vécu, tout au début au Mexique : il y avait les maires, les vaqueros, les propriétaires, des scientifiques qui discutaient sur : «Comment fait-on?», « quels sont les objectifs de gestion de conservation? », « comment organise-t-on l'espace pour assurer ces différents objectifs de formation, d'éducation, de conservation de la biodiversité? ». On parlait des ressources génétiques, de diversité écologique, ou de ressources biologiques tout court, et puis de la façon d'engager des activités d'écodéveloppement. Dès le départ, il y avait donc une implication des populations concernées, et bien évidemment il y avait une vision d'écologie. Et forcément une incitation par les chercheurs et le directeur de l'Institut national d'écologie de Mexico, le Professeur Gonzalo Halffter, qui allaient discuter avec les paysans pour les intéresser au projet. Ils adoptaient l'idée ou non, mais ils construisaient quelque chose ensemble. Au départ, il y avait bien sûr aussi une sélection des territoires, c'est-à-dire faite par les écologistes qui considèrent la planète, la biosphère, les grands types d'écosystèmes, et qui disent que cela serait bien d'avoir un désert au nord du Mexique. L'idée sous-jacente est que la planète est faite de grands écosystèmes, et que, même si on les transforme, et on les transforme de plus en plus, c'est la base même du fonctionnement de la biosphère. Avoir ciblé l'ensemble des écosystèmes de la planète et distinguer des lieux particulièrement intéressants par leur patrimoine naturel et leur patrimoine culturel, parce que l'on s'efforce de les associer, c'était un progrès considérable. En 1995, à l'occasion de la conférence de Séville où un point a été fait sur les réserves de biosphère, on recensait quelque 380 réserves de biosphère. Aujourd'hui, c'est plus de 480, ce qui veut dire aussi qu'il y a une dynamique : l'idée avance, séduit. Cela dit, aucun bilan rigoureux entre ce que l'on affiche et ce qui est réalisé n'est disponible à ma connaissance, et c'est fort dommage car l'effet d'exemplarité (éventuelle) ne joue pas.

Prenons les réserves de biosphère françaises : lorsque j'ai commencé à m'intéresser au programme MAB, j'avais été déçu par certaines d'entre elles, parce qu'il y avait plusieurs critères qui n'y étaient pas vraiment respectés - notamment la faible intégration de la recherche. Mais quand on songe au fait que ces idées étaient assez fortement nouvelles, et que leur mise en œuvre sur le terrain $\mathrm{n}^{\prime}$ avait rien de facile, tout cela peut se comprendre.

La mise en musique passe par des structures qui doivent obéir aux règles juridiques des pays concernés. Une réserve de biosphère doit donc être portée par quelque chose. En France, ces structures sont, soit des parcs naturels régionaux ou des parcs nationaux quand ils existent, soit d'autres structures quand il n'y a ni l'un, ni l'autre. Il pouvait donc y avoir des ambiguïtés, parce que les objectifs et les contraintes, par exemple du parc national à l'ancienne, à la française, n'étaient pas forcément tout à fait compatibles avec la philosophie des réserves de biosphère. Les parcs naturels régionaux, eux, sont beaucoup plus souples, il était plus facile de trouver 
un accommodement avec des objectifs de réserves de biosphère ${ }^{5}$.

On a l'impression d'un certain bricolage dans la mise en œuvre et non d'une véritable politique. Les services du ministère de $\mathrm{l}^{\prime}$ Environnement avaient du mal à maîtriser ça, à assumer simultanément plusieurs types de structures. Ainsi, mal relayé sur le terrain avec, des moyens insuffisants de surcroît, il ne faut pas s'étonner que cela n'ait pas bien marché. Donc, globalement, le bilan n'est sûrement pas terrible si on veut des choses quantifiées. Cependant, avec le recul de l'expérience - je suis allé de nombreuses fois sur le terrain - le seul fait qu'il y ait eu des débats est déjà très intéressant. Dans les réserves de biosphère, il y a des comités de gestion et des conseils scientifiques, avec des échanges entre les deux. Même si ça ne progresse pas beaucoup, le fait de rassembler autour de la table, des chasseurs, des pêcheurs, des instituteurs, des maires qui discutent de leurs problèmes et de leurs conflits, ça n'a l'air de rien, mais ça peut permettre d'avancer sérieusement sur ce que l'on appelle aujourd'hui le développement durable et la conservation participative. Dans ce contexte, je pense que, si le bilan du MAB n'est pas vraiment prestigieux, c'est que les moyens qui y ont été mis furent insuffisants. Quand il s'agit de modifier des équilibres, d'intervenir dans des enjeux économiques et sociaux, d'agir sur l'éducation, il faut des moyens plus lourds que ce qui a été mis jusquelà - et cette critique va pour l'ensemble de la protection de la nature. Mais néanmoins, ce concept « réserve de biosphère » est quelque chose d'utile, voire d'essentiel comme je l'ai dit à la conférence de Paris, à l'Unesco en janvier 2005. Des réserves de biosphère existent dans plus de 100 pays. Certaines sont probablement fictives, mais il faut bien réaliser que c'est le seul dispositif qui soit commun à l'ensemble des pays de la planète : on peut parler en France de réserves biologiques intégrales, de parcs nationaux, de parcs régionaux, mais dans tous les pays du monde, le seul dispositif partagé est celui des réserves de biosphère : il obéit à une conception d'ensemble, qui a été raisonnée et discutée à l'échelle internationale. Qui plus est, ce dispositif est organisé en réseau. Il y a les réseaux nationaux, qui marchent évidemment en fonction de ce que valent les gens qui les animent et des moyens dont ils disposent, mais cela existe. C'est aussi organisé en réseau à l'échelle mondiale, sorte d'organisation planétaire de la diversité de la biosphère. Je trouve que c'est un dispositif intéressant. Qui plus est, il a une vertu majeure : une réserve de biosphère est sans frontières, car c'est un programme d'action plus qu'un territoire sensu stricto. Un parc national a une frontière, et quand on l'a franchie, les autorités qui gèrent le parc n'ont plus rien à dire. Une réserve de biosphère, c'est un projet de territoire et

\footnotetext{
${ }^{5}$ NDLR, $c f$. Cibien, C., 2006. Les réserves de biosphère : des lieux de collaboration entre chercheurs et gestionnaires en faveur de la biodiversité, Natures Sciences Sociétés, 14, 1, 84-90.
}

d'action, avec une zone centrale ou plusieurs zones centrales, une zone tampon puis une zone périphérique qui n'est pas bornée.

Aujourd'hui, on parle de réserve de biosphère à propos de villes, de régions fortement urbanisées. Cela permet réellement de dire, oui il y a des zones éventuellement exemplaires, où il y a plus de richesses biologiques, où on fait un effort particulier de sauvegarde de milieux, mais il y a aussi des zones ordinaires où on s'intéresse à la biodiversité et qui ont besoin d'être aménagées. L'aménagement et la façon de vivre dans ces territoires peuvent impliquer des préoccupations concernant la nature qui $s^{\prime} y$ trouve, fusse-t-elle ordinaire.

Le concept lui-même et la révision du concept qui a été rendu plus contraignant d'ailleurs ont été actualisés à Séville, dans un petit document remarquable intitulé «La Stratégie de Séville». Par rapport à notre discussion sur la biologie de la conservation et l'interdisciplinarité, je trouve que c'est beaucoup plus pragmatique : cela part de l'objectif de créer des conditions de développement durable à l'échelle régionale et d'utiliser les réserves de biosphère comme des espaces où les gens expérimentent un développement durable qu'ils définissent eux-mêmes.

P.M. : Finalement le concept de réserve de biosphère ne recoupe-t-il pas pour une part le dispositif réglementaire français de parc naturel régional?

Robert Barbault : Oui, la notion de parc naturel régional est tout à fait appropriée pour mettre en œuvre ce concept. D'ailleurs quand on regarde où ça marche, $c^{\prime}$ est souvent quand un parc naturel régional est support, car leur conception se rapproche de celle qui préside aux réserves de biosphère. Les pouvoirs publics ont révisé la loi sur les parcs nationaux, révision qui s'inspire du statut des réserves de biosphère. Cela va donc dans le bon sens. Certes les intégristes de la conservation pensent trouver à redire à ces structures qui prônent des objectifs de développement durable.

M.R. : Il y a peut-être d'un côté un intégrisme, comme vous le mettez en avant, mais de l'autre côté, on pourrait avoir la crainte de projets qui restent seulement sur le papier, avec beaucoup de belles idées et de bons principes. Comme vous le soulignez, a-t-on les moyens ensuite de les réaliser et peut-on demander aux populations, aux institutions qui sont déjà débordées par les nombreuses demandes de tous les échelons étatiques, européens, de rajouter une couche institutionnelle?

Robert Barbault : Oui, c'est une question juste, mais précisément, je vais plus loin dans ma prétention, dans la mesure où le concept de réserve de biosphère, qui est très souple, permet d'y mettre tout le reste. On peut s'en servir de cadre général, un cadre conceptuel avec des objectifs ambitieux et ensuite des éléments concrets : NATURA 2000, les ZNIEFF (Zones naturelles d'intérêt écologique, 
faunistique et floristique), peuvent être gérées dans cet espace-là, avec une même vision d'ensemble. C'est un pari audacieux, parce que c'est une entité qui est définie internationalement. Mais une telle dynamique existe en Espagne : la Catalogne souhaitait faire des Pyrénées une réserve de biosphère partagée avec la France et en y mettant des moyens. Force est de constater que du côté français c'est beaucoup plus difficile. C'est vrai que la question des moyens est importante. Et sur ce plan il faut dire que les causes qui conduisent à l'érosion de la biodiversité sont les mêmes que celles qui aboutissent à la pauvreté, au chômage : ce sont les symptômes d'un monde qui fonctionne mal. Si l'on parle de «développement durable », cela veut dire que l'on a compris que le développement tel qu'on l'avait vécu jusque-là ne pouvait pas durer, qu'à le poursuivre on allait droit dans le mur.

\section{M.R. : Belle utopie?}

Robert Barbault : La vraie utopie, c'est de se contenter de paroles sans analyser pourquoi le développement qu'on a aujourd'hui n'est pas durable. La question, ce n'est pas forcément de savoir ce qu'on peut faire, c'est d'abord de formuler un diagnostic correct, et de le faire partager, d'échanger avec tous les citoyens concernés, $\mathrm{d}$ 'identifier ensemble les problèmes et en parler : c'est un progrès important. Peut-être est-ce mon côté moralisateur; mais si l'homme est une espèce exceptionnelle, producteur de créations artistiques, de philosophie, capable de gouverner le monde, de planifier son avenir, on devrait alors être capable de mobiliser les ressources nécessaires pour que nos enfants et nos petits enfants vivent dans des conditions décentes!

Quand on veut nous faire croire qu'on va résoudre les problèmes d'aujourd'hui en allant coloniser la planète Mars, ou une autre, pour y trouver les ressources dont on aura besoin, je m'interroge : si on n'est pas capable de résoudre les problèmes qui se posent aujourd'hui sur cette planète, pourquoi cela serait-il possible sur une autre, fort inaccessible de surcroît. Je ne dis pas qu'en termes d'exploration scientifique, ça ne soit pas à faire, mais laisser croire comme le font certains collègues, y compris de très grands " médiatiques », que l'on va résoudre nos problèmes ainsi, c'est franchement irritant. Donc je ne crois pas qu'un développement humain durable soit impossible : c'est seulement difficile parce que premièrement, on n'en discute pas, et que deuxièmement, on n'en fait pas l'analyse sur le fond. On pourra améliorer progressivement la situation. À l'échelle d'un pays comme la France, les problèmes ne sont pas aussi considérables que ça. On a une démographie qui s'est stabilisée; on a des milieux diversifiés dont certains sont bien gérés depuis longtemps ; enfin, on a des capacités de concilier des besoins de développement raisonnables, intelligemment conçus, avec des préoccupations de sauvegarde de la diversité des milieux et des espèces autant qu'il est possible.

Là où c'est évidemment plus difficile, c'est dans les pays à populations très denses, avec un sousdéveloppement marqué, et où il faudrait apporter des moyens pour permettre un redressement : la façon dont fonctionne le Monde ne permet pas ça. Les choses sont telles qu'on ne voit pas, quand on vit à Paris par exemple, que la qualité de vie dont on bénéficie dépend de tas de choses qui se passent dans le Monde entier. Avec des tas de conséquences positives et négatives, en termes d'utilisation de l'énergie, de transports.

P.M. : Ce que j'ai retenu de vos propos et de nos échanges, c'est que les réserves de biosphère sont des lieux de dialogue dans les différents pays du Monde, de différentes cultures, de différentes conditions économiques, etc., et qu'à partir de là, on peut aborder de façon concrète avec les populations locales l'intérêt de conserver la biodiversité et par effet de tâche d'huile, d'avancer de façon générale...

Robert Barbault : Je considère en effet les réserves de biosphère comme un concept, comme un projet. J'ai dû écrire cela dans un article pour la Guyane au moment où on parlait du projet de parc national : si on était audacieux, on décréterait que la Guyane tout entière est une réserve de biosphère.

M.R. : Je partage votre intérêt sur l'idée de partage, mais je ne suis pas aussi optimiste, j'ai quand même le sentiment, en particulier en France, que la hiérarchie et le système bureaucratique sont tellement forts que par exemple, je vois rarement des représentants du paysan de base dans les réunions où l'on prend des décisions. On peut voir le maire ou le président d'un puissant syndicat qui éventuellement va avoir une voix consultative, mais il est extrêmement rare d'avoir les pêcheurs de l'estuaire, les bergers de la montagne, ou d'autres qui ont pourtant des pratiques, et des savoirs, avoir une voix dans ces organismes, qu'ils soient parcs nationaux, parcs naturels régionaux, ou réserves de la biosphère.

Robert Barbault : Cela, c'est très juste, mais dans les parcs qui marchent bien, il y a normalement des activités de communication, des conférences publiques, et donc des possibilités d'implication de quiconque le souhaite.

M.R. : Même si on en a déjà un peu parlé, pouvez-vous nous faire part de vos réflexions sur l'interdisciplinarité, à partir d'expériences récentes personnelles ou proches de vous, qui mettent en avant la nécessité d'analyser et de planifier la conservation et la gestion en interdisciplinarité. Lesquelles? Comment?

Pensez-vous qu'on en est déjà là, c'est-à-dire à la mise en commun d'un objet de recherche complexe en amont d'un programme, qui serait une véritable 
interdisciplinarité, ou n'en est-on encore qu'à une pluridisciplinarité ? Ce qui pose alors le problème d'intégration : dans les diverses disciplines on n'a pas les mêmes échelles, ni de temps, ni d'espaces et à la fin la juxtaposition des résultats est une vision kaléidoscopique.

J'ai le sentiment que dans d'autres pays que la France, les sciences humaines ont plus droit à la parole que chez nous dans le domaine de la gestion de la biodiversité. Quelles sont les sciences et les disciplines qui vous semblent particulièrement pertinentes et qui déjà vous semblent capables de travailler avec l'écologie?

Robert Barbault : Il faut déjà commencer par définir les objectifs de gestion, avant de définir les projets de recherches qui y sont liés. Cela suppose que les conseils scientifiques de ces parcs soient multidisciplinaires au départ, et dans la pratique, ce qu'on a vécu, c'est de la pluridisciplinarité. En France, il n'y a pas d'interdisciplinarité effective. Mais on sent qu'il y a de plus en plus d'échanges entre disciplines, on sent que ça progresse. Quand on regarde comment fonctionnent les différents parcs naturels régionaux, par exemple le parc naturel régional des Vosges du Nord, qui est en même temps une réserve de biosphère, on se dit que les choses évoluent dans le bon sens. Mais au départ, il y a eu un diagnostic interdisciplinaire ${ }^{6}$.

C'est vrai que dans les structures françaises (parcs nationaux ou autres) les conseils scientifiques ont été généralement très dominés par les naturalistes, en dépit parfois de la volonté des responsables de parcs. Au MAB, on insiste lourdement sur la nécessité de concevoir dès le départ des conseils scientifiques équilibrés. C'est une exigence qui est clairement posée. Et elle est mise en œuvre, même si cela a suscité quelques difficultés avec certains naturalistes purs et durs.

M.R. : Pour l'introduction ou la réintroduction d'une espèce par exemple, a-t-on besoin d'équipes interdisciplinaires?

Robert Barbault : On aurait besoin d'équipes interdisciplinaires. Les gens peuvent y travailler séparément, mais sur le même "système », en étant au courant de ce que font les uns et les autres. Mais il ne s'agit pas d'aboutir à une science nouvelle qui intégrerait les autres : ce n'est pas nécessaire puisqu'on fait dans le pragmatique.

M.R. : Moi, je ne pensais pas à une science nouvelle, comme vous le dites, parce que c'est une grosse ambition, et à la limite ça serait à nouveau une compartimentation, mais par contre, peut-être à un peu plus que travailler chacun de son côté. Dans la mesure où je crois que c'est en amont qu'il faut construire un objet de recherche commun, et que chaque discipline accepte de se mettre au service de l'autre, même si ce n'est pas le centre de votre intérêt, nous, nous aurions besoin que vous fassiez ça et réciproquement; ça me semble important, que chacun ne travaille pas de son côté.

Robert Barbault : Je suis d'accord, là est l'objectif à atteindre. C'est ce que je plaide auprès du MEDD (ministère de l'Écologie et du Développement durable), mais aussi auprès du ministère de la Recherche. Pour moi la priorité, ce sont des projets de recherche qui impliquent des chercheurs de différentes disciplines et des gestionnaires, sur des objectifs d'intérêt commun pour les deux. C'est cela que le ministère de l'Écologie et du Développement durable devrait soutenir sur les applications des sciences de la conservation, avec au besoin un soutien du ministère de la Recherche. Je ne demande pas au MEDD de tout financer, mais je lui dis que sa mission c'est cela, entraîner le ministère de la Recherche sur un programme de recherche qui privilégierait: (1) l'implication conjointe d'acteurs de la recherche et d'acteurs de la gestion des espaces ; (2) le développement d'approches interdisciplinaires, voire transdisciplinaires incorporant notamment les approches des sciences de l'Homme et de la société ; (3) la prise en compte d'un contexte marqué par les changements globaux (changements climatiques y compris) ; et (4) une ambition d'observatoire de recherche sur la dynamique de la biodiversité conçu sur le principe de réseaux - et qui ferait du réseau des espaces protégés un grand instrument de recherche et de formation pour la conservation de la nature.

\footnotetext{
${ }^{6}$ NDLR : cf. Génot, J.-C., 2003. L'interdisciplinarité à l'épreuve de la pratique : l'exemple d'un espace protégé, Natures Sciences Sociétés, 11, 4, 413-418.
} 